\title{
Influence of COVID-19 Pandemic on Endoscopic Procedures in Two European Large-Capacity Endoscopy Units: “Keep Calm, Keep Safe and Scope on?"
}

\author{
Michal Zorniak ${ }^{a, b}$ Simon Sirtl ${ }^{b} \quad$ Ujjwal Mukund Mahajan ${ }^{b}$ \\ Hans-Christian Stubbe ${ }^{b}$ Mateusz Chapula ${ }^{a}$ Piotr Wosiewicz ${ }^{a} \quad$ Marek Hartleb $^{a}$ \\ Julia Mayerle Christian Schulz $^{b}$ \\ aDepartment of Gastroenterology and Hepatology, Medical University of Silesia, Katowice, Poland; bepartment of \\ Medicine II, University Hospital, LMU Munich, Munich, Germany
}

\section{Take Home Message}

- The COVID-19 pandemic forced fundamental changes to the management of endoscopic procedures.

- As a result of pandemic-related restrictions, the overall number of endoscopic procedures was significantly reduced and restricted to essential procedures.

- Besides a reduced overall number a significant increase in therapeutic procedures was noted and in tertiary centers, the number of complex endoscopic procedures remained stable.

- Personal safety measures established in endoscopy proved to be efficient and safe in preventing SARSCoV2 infection of staff or spreading in endoscopy units.

- During the COVID-19 pandemic endoscopy, teaching had to be neglected and solutions allowing fellows to continue training are urgently needed

\section{Keywords}

COVID-19 pandemic · Endoscopy procedures · Pandemic restrictions · Quality in endoscopy · Endoscopy training

\begin{abstract}
Background: The COVID-19-pandemic poses challenges to the medical system and especially to endoscopic staff and patients. National, European and International societies provided recommendations on how to safely perform endoscopic procedures during the current pandemic. Until now, the effect of the current pandemic on tertiary endoscopy centers has not been reported. Objective: The aim of this
\end{abstract}

was to analyze the influence of the early SARS-CoV2-pandemic on endoscopic care and work flow in 2 European tertiary endoscopy units. Methods: Data from 2 tertiary endoscopy units (Katowice and Munich) were retrospectively collected during the early pandemic and compared to an equivalent pre-pandemic period. Data include procedures, complications, benchmarks, and influence on endoscopy training. Results: During the early pandemic, we noted a highly significant decrease (49.1\%) in the overall number of all endoscopies with a significant increase in therapeutic procedures. Besides, there were no significant differences in

M.Z. and S.S. contributed equally to the manuscript. 
the number of urgent endoscopic retrograde cholangiopancreatography or interventional endoscopic ultrasound procedures. The exceptional situation reduced endoscopic procedures performed by trainees significantly. Conclusions: The SARS-CoV2-pandemic halved the endoscopy service of 2 tertiary centers while maintaining an urgent therapeutic service. Recommended personal safety measures in endoscopy proved to be efficient and safe in preventing SARSCoV2 infection of staff or spreading. Unnecessarily, the SARS$\mathrm{CoV} 2$ pandemic prevented routine endoscopy training.

(c) 2020 S. Karger AG, Basel

\section{Introduction}

The COVID-19 pandemic poses new challenges to endoscopy units. Reducing scheduled routine procedures in combination with a well-implemented triage system became a permanent feature of clinical reality. Already at the early stage of the pandemic the American Gastroenterology Association (AGA), as well as joint European Society of Gastrointestinal Endoscopy (ESGE) - European Society of Gastroenterology and Endoscopy Nurses and Associates (ESGENA) independently from rules and regulations implemented by local government, released official statements giving guidance on endoscopic procedures $[1,2]$.

Endoscopic interventions should be performed in patients who substantially benefit, and postponement is not feasible [3]. A recent analysis from New York City demonstrated that new pandemic-related restrictions caused a drastic reduction of overall endoscopic procedures and procured fundamental changes in the processes of endoscopy units [4]. A second report on the influence of the pandemic on endoscopy was published recently by an Austrian group analyzing 3 weeks before and after national lockdown. Surprisingly, this study showed a dramatic (40.7\%) decrease in diagnosis of upper gastrointestinal bleeding events countrywide [5]. Nevertheless, until now, the data on real-life effects of pandemic-related restrictions on gastrointestinal endoscopy is scarce.

The ESGE-ESGNA statement on gastrointestinal endoscopy and the COVID-19 pandemic, which was published on the last day of our observation (April 17, 2020), suggested that future research on this topic should be enforced and include [2]:

- How did COVID-19 affect the endoscopy unit's workflow?

- How did COVID-19 affect fellows' training?

- How was the GI endoscopy organized in terms of care for patients?

Influence of COVID-19 Pandemic on

Endoscopic Procedures
Although most of the international societies published their recommendations, little is known about the real-life influence of early phase COVID-19 pandemic on the functioning of large-capacity endoscopy centers. Until May 10, 2020, about 16,000 people in Poland and more than 170,000 people in Germany were confirmed positive for COVID-19 based on an RT-PCR positive result [5]. Through co-operation between 2 large-capacity, central European endoscopy units (Department of Medicine II, LMU, Munich, Germany, and Department of Gastroenterology and Hepatology, Medical University of Silesia in Katowice, Poland) we analyzed the changes on medical care in the 2 endoscopy units during the pandemic. Here, we present in line with the ESGE-ESGNA recommendations data on the influence of COVID-19 related changes on endoscopic procedures: gastroscopy, colonoscopy, endoscopic retrograde cholangiopancreatography (ERCP), and endoscopic ultrasound (EUS), including the changing role of endoscopy fellows within this workforce demanding time.

\section{Material and Methods}

We retrospectively collected data on endoscopic procedures from the Department of Medicine II, University Hospital, LMU, Munich, Germany, and the Department of Gastroenterology and Hepatology, Medical University of Silesia, Katowice, Poland. Both departments included in the study belong to the group of largest endoscopy-centers in Bavaria and Silesia, respectively. Based on lo$\mathrm{cal}$ and national reports, those regions belonged to the most heavily affected by the pandemic in their countries (Bavaria: 25\% of overall cases in Germany and Silesia: 22\% of overall cases in Poland) [6].

Data about endoscopic procedures were collected from endoscopy unit databases in both centers. In the next step, data from both centers were anonymized to build an overall database used for statistical comparisons. Incomplete data on procedures, for example, information about indications for the procedure (outpatient/inpatient/urgent/emergency) or trainee-assistance, were excluded from the study.

To evaluate the influence of the COVID-19 pandemic on endoscopy units, we compared a timeframe between March 16, 2020, and April 17, 2020, in Germany and Poland. It reflects an early phase of the crisis, similarly in both countries, with regards to the declaration of a pandemic by the World Health Organization [7]. We compared this time frame with the corresponding period the year before (March 25, 2019-April 26, 2019, different dates to account for the same number of working days and avoid bias caused by school holidays). Both compared periods included the same number of weekend days ( 8 each) and holidays (2 each).

Before qualification for endoscopic procedures, patients were screened following current local COVID-19 recommendations. In the early phase of the pandemic, this included a careful history and physical examination with additional PCR or imaging, primarily if the infection was suspected. In both centers, the number of assistant staff was based on national guideline recommendations and 
Two large-capacity academic endoscopy units

Munich (Germany) / Katowice (Poland)
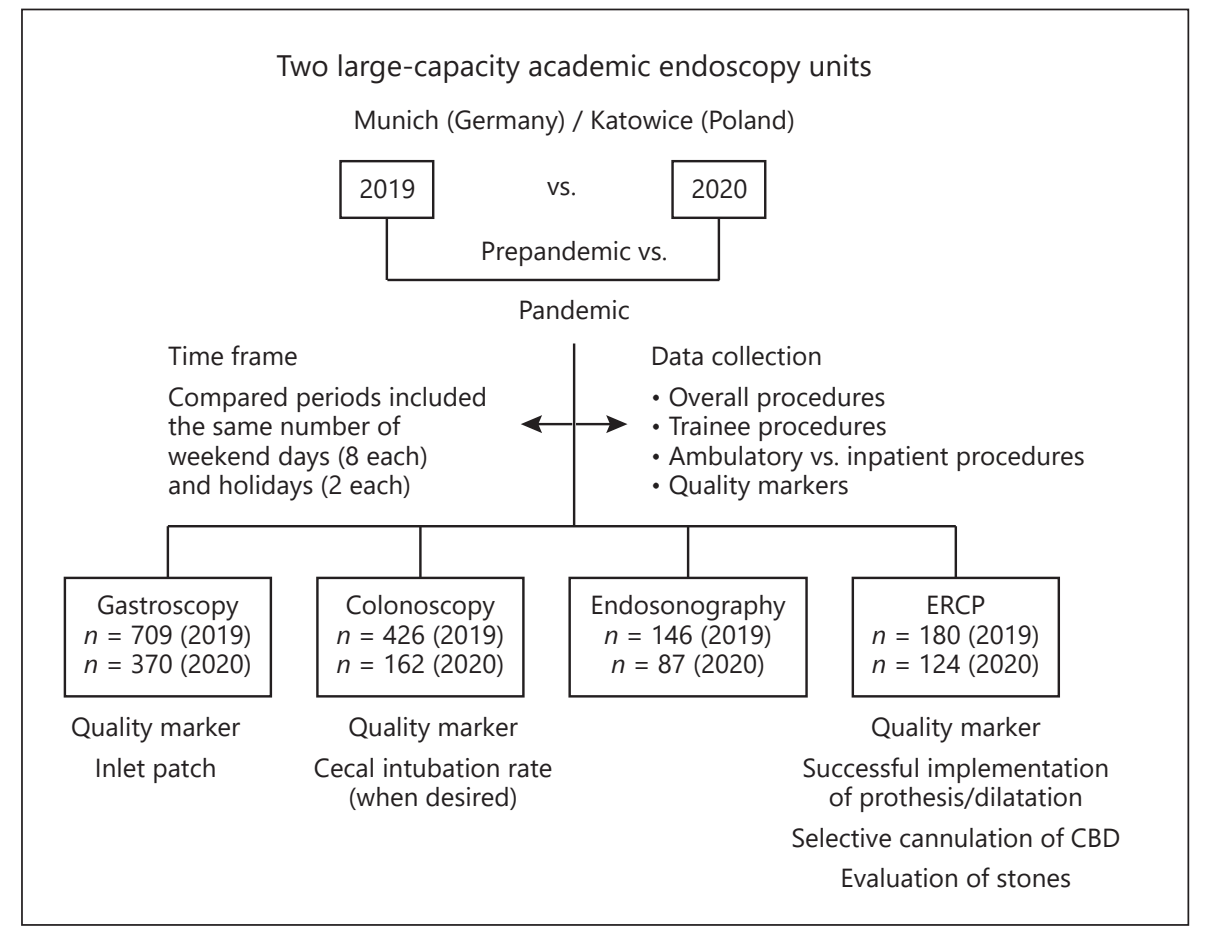

Fig. 1. Comparison of pre-pandemic and pandemic functioning of large-capacity endoscopy units. Flowchart of patient selection for the study periods (March 25, 2019April 26, 2019 vs. 16.03.2020-17.04.2020). Periods compared included the same day of holiday and weekend days. was not influenced by the pandemic. Each physician and assistant staff involved worn disposable PPE: FFP2 masks, protective glasses, gloves, and garments, as recommended by the national authorities. None of the patients qualified for endoscopic procedure was diagnosed positive for COVID-19 at the time point of examination in both centers. However, in 2 patients, SARS-CoV2-infection was diagnosed later in Munich.

\section{Procedures Analyzed in the Study}

We collected data on endoscopic procedures such as gastroscopy, colonoscopy, ERCP, and EUS. We documented an overall number of procedures, types of procedures (outpatients/inpatients/urgent/emergency), benchmarks of endoscopic procedures, and influence on endoscopy trainee's involvement in procedures (for details, see Fig. 1).

Interventional gastroscopy procedures included all upper GI endoscopies performed with the intention of treatment, that is, upper GI-polypectomy, upper GI-tract dilatation, routine esophageal variceal ligation, percutaneous endoscopic gastrostomy, and urgent treatment for bleeders. ERCP procedures were defined as urgent when performed $<24$ h of patients' hospital admission.

\section{Endoscopy-Performance Indicators}

To further evaluate the potential influence of restrictions related to COVID-19 pandemic on the performance of endoscopic procedures, we compared available generally accepted performance indicators with the endoscopic results during a comparable pre-pandemic period:

- Gastroscopy:

- Inlet patch detection rate: based on available data, the prevalence of inlet patches varies between $0.18 \%$ in retrospective studies (Neumann et al. [8], a study of 487,229 unique patients with biopsy-proven inlet patches) up to $11.7 \%$ in selected studies in which inlet patches were actively searched [9]. Despite mentioned differences, reporting on the inlet patch detection rate is recognized as a performance-marker in upper GI-endoscopy [10].

- Colonoscopy:

Cecal intubation rate (CIR) is one of the most well-defined and worldwide accepted performance indicators used to assess the quality of colonoscopy [11]. Low CIR is closely correlated with a low adenoma detection rate and an increased risk of post-colonoscopy colorectal cancer. CIR is not only a performance indicator but also reveals the endoscopic skills of a physician. Experienced colonoscopists have been shown to intubate the cecum in more than $90 \%$ of cases [12].

- ERCP:

Selective bile duct cannulation rate (native papilla), appropriate stent placement in patients with biliary obstruction below the hilum, bile duct stone extraction: ESGE suggests that selective biliary cannulation rate of a native papilla should be achieved in $80 \%$ of cases when using standard cannulation techniques [13]. ESGE suggests that appropriate stent placement in patients with biliary obstruction below the hilum, and bile duct stone extraction should be achieved in 95 and 90\%, respectively [14]. Modifying these recommendations, we included data concerning all biliary interventions in case of stenosis below the hilum, including also successful dilatations and successful implementation of a stent in case of irremovable biliary stones.

\section{Statistical Analysis}

We compared demographic characteristics of participants in univariate and multivariate analyses. Analyses were weighted and performed using $\mathrm{n}$ R (Version 3.5.2) and R studio (Version 1.1.442, 
Table 1. General comparison of patients' age and sex as well as procedures-cohorts in pre-pandemic and pandemic periods. ERCP ; EUS

\begin{tabular}{llll}
\hline & 2019 & 2020 & \\
\hline Age & $59( \pm 16$ yr $)$ & $58( \pm 11 \mathrm{yr})$ & NS \\
Sex (Female) & $n=681 / 1461(46.6 \%)$ & $n=320 / 743(43 \%)$ & NS \\
Overall procedures & $n=1,461$ & $n=743$ & $-49.2 \%$ \\
$\quad$ Gastroscopy & $n=709$ & $n=370$ & $-48 \%$ \\
$\quad$ Colonoscopy & $n=426$ & $n=162$ & $-62 \%$ \\
$\quad$ ERCP & $n=180$ & $n=124$ & $-32 \%$ \\
EUS & $n=146$ & $n=87$ & $-41 \%$ \\
\hline
\end{tabular}

ERCP, endoscopic retrograde cholangiopancreatography; EUS, endoscopic ultrasound.

Table 2. The influence of COVID-19 pandemic on overall and trainee-performed procedures in two high capacity central-European endoscopy centers

\begin{tabular}{|c|c|c|c|c|}
\hline & 2019 & 2020 & & $\begin{array}{l}\text { Baseline differences } \\
\text { between the centers }\end{array}$ \\
\hline \multicolumn{5}{|l|}{ Gastroscopy } \\
\hline Overall procedures & $n=709$ & $n=370$ & $-48 \%$ & - \\
\hline Trainee procedures & $245 / 709(34.6 \%)$ & $101 / 370(27.3 \%)$ & $p=0.015$ & NS \\
\hline \multicolumn{5}{|l|}{ Colonoscopy } \\
\hline Overall procedures & $n=426$ & $n=162$ & $-62 \%$ & - \\
\hline Trainee procedures & $208 / 426(48.8 \%)$ & $28 / 162(17.3 \%)$ & $p \leq 0.001$ & NS \\
\hline \multicolumn{5}{|l|}{$E R C P$} \\
\hline Overall procedures & $n=180$ & $n=124$ & $-32 \%$ & - \\
\hline Trainee procedures* & $63 / 87(72.4 \%)$ & $35 / 73(47.9 \%)$ & $p \leq 0.001$ & Munich only* \\
\hline \multicolumn{5}{|l|}{ EUS } \\
\hline Overall procedures & $n=146$ & $n=87$ & $-41 \%$ & - \\
\hline Trainee procedures* & \multicolumn{4}{|c|}{ No EUS training program in compared periods } \\
\hline
\end{tabular}

ERCP, endoscopic retrograde cholangiopancreatography; EUS, endoscopic ultrasound. * There was no EUS, training program in neither of included centers and no ERCP, training program in Katowice.

Boston, USA). For the univariate analyses, categorical variables were compared using the Wald-adjusted Pearson $\chi^{2}$ test with Yate's correction. Comparisons were represented with prevalence odd's ratio (POR) with a 95\% confidence interval (CI). $p<0.05$ was considered statistically significant.

\section{Results}

Patients' characteristics are summarized in Table 1. During the pandemic, there was a notable decrease in the overall number of all procedures as follows: gastroscopy $(-48 \%)$, colonoscopy $(-62 \%)$, ERCP $(-32 \%)$, and EUS (-41\%). COVID-19 pandemic has also profoundly influenced training in the endoscopy units, causing a significant decrease in the number of all trainee-performed procedures (for details, see Tables 2 and 3). There was a significant increase in gastroscopic - inter- ventional procedures in comparison to procedure with diagnostic intentions ( 16.8 vs. $25.6 \%$, respectively, $\chi^{2}=$ $10.217, d f=1, p=0.001$; OR [CI 95\%]: 1.664 [1.2252.261]). Interestingly, we noted significantly different local strategies in dealing with outpatients' procedures (for details, see Table 4 and online suppl. Material; for all online suppl. material, see www.karger.com/ doi/10.1159/000511076). In summary, this has finally led to a significant decrease of outpatients colonoscopies and gastroscopies in Munich (45 vs. $22.1 \%$; $\chi^{2}=$ $30.751, d f=1, p<0.001$ and 27.4 vs. $14.3 \% ; \chi^{2}=15.517$, $d f=1, p<0.001$, respectively) and an increase in Katowice ( 18.7 vs. $50 \% ; \chi^{2}=20.696, d f=1, p<0.001$ and 42.3 vs. $\left.54.5 \% ; \chi^{2}=4.093, d f=1, p=0.04\right)$ - for details see Table 4 . There were no significant differences in the number of urgent/emergency ERCP procedures (for details, see Tables 5, 6). 
Table 3. Comparison and trend analysis of trainee-performed endoscopic procedures before and during COVID-19 pandemic

\begin{tabular}{|c|c|c|c|}
\hline & 2019 & 2020 & Tests for trends \\
\hline $\begin{array}{l}\text { Gastroscopy } \\
\text { Trainee procedures }\end{array}$ & $245 / 709(34.6 \%)$ & $101 / 370(27.3 \%)$ & $\begin{array}{l}\chi_{\mathrm{DF}=1}^{2}=5.551, p=0.001 \\
P O R(95 \% C I): 0.711(0.539-0.937)\end{array}$ \\
\hline $\begin{array}{l}\text { Colonoscopy } \\
\text { Trainee procedures }\end{array}$ & $208 / 426(48.8 \%)$ & $28 / 162(17.3 \%)$ & $\begin{array}{l}\chi_{\mathrm{DF}=1}^{2}=47.296, p<0.001 \\
P O R(95 \% C I): 0.219(0.139-0.343)\end{array}$ \\
\hline $\begin{array}{l}E R C P \\
\text { Trainee procedures }\end{array}$ & $63 / 87(72.4 \%)$ & $35 / 73(47.9 \%)$ & $\begin{array}{l}\chi_{\mathrm{DF}=1}^{2}=9.008, p=0.002 \\
P O R(95 \% C I): 0.350(0.181-0.676)\end{array}$ \\
\hline
\end{tabular}

ERCP, endoscopic retrograde cholangiopancreatography; EUS, endoscopic ultrasound.

Table 4. Individual differences and tests for trending in out-patients' procedures in two high capacity centralEuropean endoscopy centers

\begin{tabular}{lccc}
\hline & 2019 & 2020 & Significance of differences \\
\hline $\begin{array}{l}\text { Munich } \\
\text { Outpatients'colonoscopies }\end{array}$ & $110 / 244(45 \%)$ & $23 / 104(22.1 \%)$ & $\chi^{2}=30.751, d f=1, p<0.001$ \\
\hline $\begin{array}{l}\text { Katowice } \\
\text { Outpatients'colonoscopies }\end{array}$ & $34 / 182(18.7 \%)$ & $29 / 58(50 \%)$ & $\chi^{2}=20.696, d f=1, p<0.001$ \\
\hline $\begin{array}{l}\text { Munich } \\
\text { Outpatients'gastroscopies }\end{array}$ & $129 / 470(27.4 \%)$ & $37 / 258(14.3 \%)$ & $\chi^{2}=15.517, d f=1, p<0.001$ \\
\hline $\begin{array}{l}\text { Katowice } \\
\text { Outpatients'gastroscopies }\end{array}$ & $101 / 239(42.3 \%)$ & $61 / 112(54.5 \%)$ & $\chi^{2}=4.093, d f=1, p=0.04$ \\
\hline
\end{tabular}

For more detailed comprehensive data, see online suppl. Material.

Regarding endoscopy performance parameters, there was a slight but significant increase of the percentage of successful biliary interventions (defined as a successful implementation of a stent or successful dilatation of distal CBD) in ERCP: 89.3 versus $97.6 \%\left(\chi^{2}=4.0966, d f=1\right.$, $p=0.04$; POR [95\% CI]: 7.944 [1.015-62.137]). However, comparing 2019 versus 2020, the ERCP-performance markers selective cannulation of CBD: 81.1 versus $74.7 \%$, respectively; $p=0.38$, and successful stone evacuation 89.6 versus $78.1 \%$, respectively; $p=0.277$ slightly decreased below the threshold of $80 \%$. However, this decrease did not reach statistical significance. There were no significant differences in diagnostic gastroscopy inlet patch-detection rate nor overall colonoscopy cecum intubation rate between compared periods (for details, see Table 5).
Except for the number of outpatients' colonoscopies, at baseline period (March 25, 2019-April 26, 2019), we found no significant differences in parameters between the 2 centers. Until the end of post-observation time (14 days after the last day included in this study, i.e., May 2, 2020), we also noted no procedure-related COVID-19 infection among endoscopy-dedicated staff in neither of the included centers.

The COVID-19 pandemic dramatically influences endoscopy fellows-in-training. We noted a significant decrease in trainees' involvement in all types of procedures, with the most severe drop in colonoscopy with POR (95\% CI): 0.219 (0.139-0.343) and ERCP with POR (95\% CI): 0.350 (0.181-0.676). The decrease was also significant but less pronounced in the case of gastroscopy: POR (95\% CI): $0.711(0.539-0.937)$. 
Table 5. The influence of COVID-19 pandemic on endoscopic procedures and endoscopy-quality markers in two high capacity centralEuropean endoscopy centers

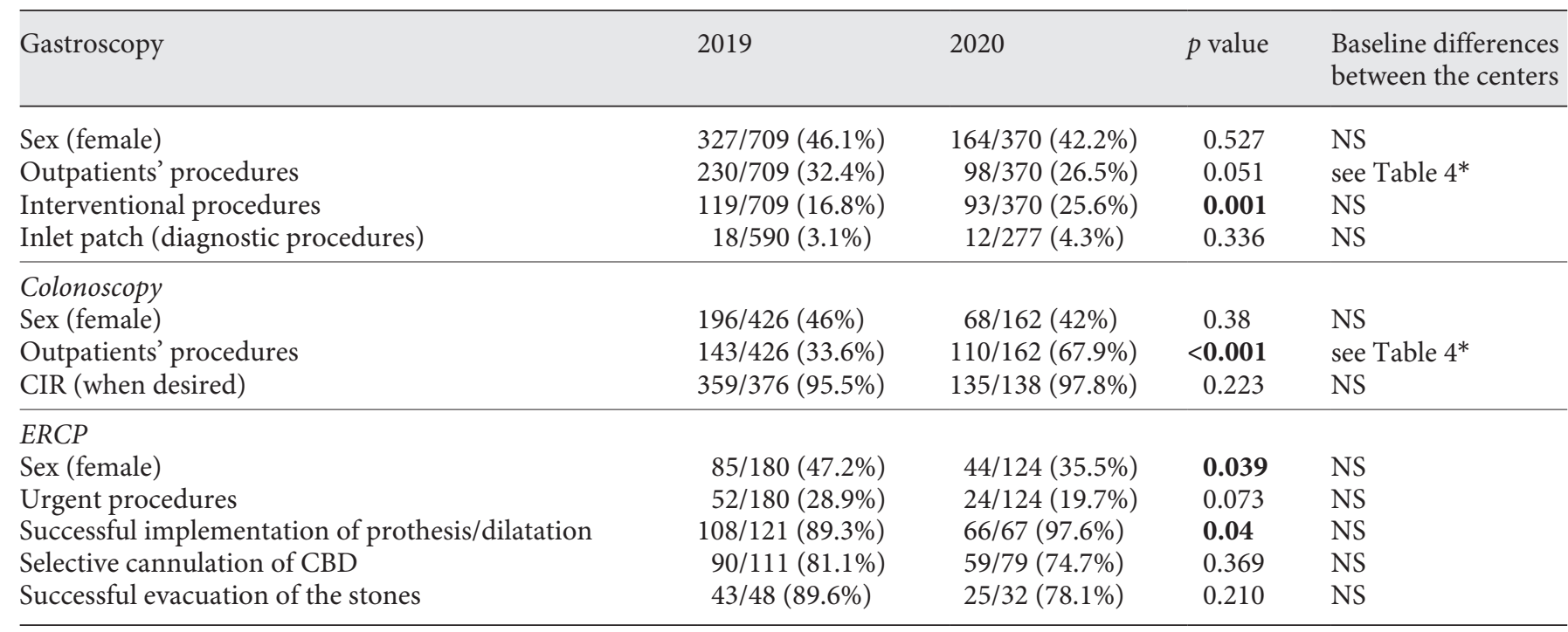

Table 6. Overall trend analysis for endoscopic procedures and endoscopy-performance markers before and during the COVID-19 pandemic

\begin{tabular}{|c|c|c|c|}
\hline Gastroscopy* & $2019, n(\%)$ & 2020 & Tests for trends \\
\hline $\begin{array}{l}\text { Gastroscopy* } \\
\text { Interventional procedures }\end{array}$ & $119 / 709(16.8 \%)$ & $93 / 370(25.6 \%)$ & $\begin{array}{l}\chi_{\mathrm{DF}=1}^{2}=10.217, p=0.001 \\
\text { POR }(95 \% \mathrm{CI}): 1.664(1.225-2.261)\end{array}$ \\
\hline $\begin{array}{l}\text { Colonoscopy* } \\
\text { Outpatients' procedures }\end{array}$ & $143 / 426(33.6 \%)$ & $110 / 162(67.9 \%)$ & $\begin{array}{l}\chi_{\mathrm{DF}=1}^{2}=5.551, p=0.001 \\
\text { POR }(95 \% \mathrm{CI}): 0.711(0.539-0.937)^{*}\end{array}$ \\
\hline $\begin{array}{l}E R C P \\
\text { Successful implementation of prothesis/dilatation }\end{array}$ & $108 / 121(89.3 \%)$ & $66 / 67(97.6 \%)$ & $\begin{array}{l}\chi_{\mathrm{DF}=1}^{2}=4.096, p=0.04 \\
\text { POR }(95 \% \mathrm{CI}): 7.944(1.015-62.137)\end{array}$ \\
\hline
\end{tabular}

ERCP, endoscopic retrograde cholangiopancreatography; CIR, cecal intubation rate; POR, prevalence odd's ratio; CI, confidence interval. * Summarized overall data for both included centers. For individual details and differences, see Table 4 and online suppl. Material.

\section{Discussion}

The COVID-19 pandemic has a tremendous impact on the function of medical facilities worldwide. Despite the early reaction of national and regional authorities and exceptional restrictions implemented, Bavaria, as well as Silesia, belonged to the most affected regions in their countries, with an exponential increase in infections (number of patients diagnosed with COVID-19 by the end of our observation was 40,615 and 1,556, respectively).

Influence of COVID-19 Pandemic on Endoscopic Procedures
Most GI endoscopy units in Europe faced shortages of personnel and PPEs, substantial reductions in the volume of screening endoscopy procedures, regulatory restrictions on prioritizing endoscopic procedures, and postponing many procedures for at least 3 months depending on the course of pandemic without knowing when patients will be rescheduled [2]. Especially academic tertiary endoscopy units are presently dealing with the novel challenge of combining clinical care, research, and fellow training under pandemic circumstances. Therefore, reallife observations allow us to evaluate the actual impact of 
the early phase of COVID-19 pandemic on 2 large-scale endoscopy centers. To date, our data are the first report of such a perspective.

As expected, the introduction of official restrictions coincided in time with a notable reduction of an overall number of endoscopy procedures. Those numbers varied dependently on type of procedure, from $32 \%$ (ERCP) to $62 \%$ (colonoscopy). It is worth to be mentioned that those numbers show a lower percentage in reduction, compared with available data from a cross-sectional survey from the New York metropolitan area, where weekly endoscopy volumes declined by $57-96 \%$ for all types of procedures [4]. As our data suggest that endoscopy procedures appear to be safe concerning endoscopy staff if precautions are adhered to, these differences could be crucial concerning collateral damage accepted in a nonscoped population.

Alongside tremendous logistic effort within hospitals to control the COVID-19 pandemic, the teaching environment changed substantially. In a recent study published by Pawlak et al. [15], the authors conducted an international survey of 770 trainees from 63 countries over 3 weeks of the pandemic. It has confirmed that $93.8 \%$ of trainees reported a decrease in endoscopy case volume. The median percentage reduction in total procedures was 99\% (interquartile range, $85-100 \%$ ), which varied internationally $(p<0.001)$ and was most significant for colonoscopy procedures. Restrictions in case volume and trainee activity were recognized as the most significant barriers.

Moreover, according to the statements of AGA and ESGE-ESGENA, published after the accomplishment of this study, only essential and fully trained personnel should be involved in endoscopy $[1,2]$. This situation, however, should not last, as training is an essential part of the functioning of a university endoscopy unit. The AGA recommendations encourage to review and determine the appropriateness of trainee involvement in procedures with consideration of procedural time. We noted a dramatic decrease in trainees' involvement in all types of procedures, with the most severe drop in colonoscopy and ERCP. This most likely reflected the necessity to relocate our fellows with advanced medical training for care in ITU or emergency department, the increasing complexity of the procedure, the need for staff safety and the reduction of number of persons exposed to possible COVID-19 infections. Nevertheless, this posed considerable dissatisfaction on our trainees which was loudly voiced. As our observation shows no endoscopy-related infections, despite minimized but maintained trainees' in- volvement, it could be carefully hypothesized that fellows previously trained in the infection prevention and control strategies for COVID-19 could safely return to performing endoscopies in a stepwise manner. This approach might involve starting from less time-consuming and more straightforward procedures to more complex ones, after appropriate COVID-19 safety training.

During the pandemic, we observed a decrease in the overall number of upper GI endoscopies but a highly significant increase in therapeutic procedures in both centers. This observation could reflect an increased focus on essential/therapeutic upper-GI endoscopic procedures due to the preselection of cases during the early COVID-19 pandemic, which was recommended by the Bavarian government and also suggested in a recently published German position paper [16]. We did not observe a significant difference in urgent ERCP procedures. This suggests that ERCP as a therapeutic procedure is essential and cannot safely be postponed without harm to the patient even under circumstances of a pandemic. However, data for comparison of this observation are not available.

Surprisingly, we noticed significant differences in handling of outpatient colonoscopy and gastroscopy procedures between centers. Although summarized data suggests that there was an overall increase in outpatient colonoscopies and a slight decrease in outpatient gastroscopies, these data are somewhat biased by the fact that both centers implemented contrary strategies. These strategies are explained by the following obstacles posed in the centers. In Munich, the government restricted elective procedures by law, while in Katowice local authorities recommended limiting patient's hospitalization whenever possible (i.e., by encouraging bowel preparation at home before colonoscopy). This resulted in an increase in outpatient procedures in Katowice while a decrease in Munich was observed (see Table 4).

Whether restrictions posed by the German government to ensure staff capacity to treat COVID-19 patients would have been necessary to prevent spreading of the infection through patients' contact within medical facilities needs to be addressed in future studies. Our data suggest that encouraging out-of-hospital self-preparation for a colonoscopy when possible, as performed in Katowice, seems to be a safe option concerning patients' and staff s safety.

Importantly, when analyzing well-established endoscopy quality benchmarks, we did not see a significant decrease in the investigated periods (see Table 5). However, during ERCP, there was a slight decrease in rate of selective cannulation and the rate of successful evacuation of 
stones, but the rate of immediate treatment success was significantly increased. Taking into consideration that those procedures were performed mostly by experienced endoscopists due to COVID-19 restrictions affecting trainees, these observations could suggest an increased level of difficulty. As an additional explanation, we would give that all procedures were done by senior endoscopists, who in the interest of time to prevent possible infection prioritized for immediate treatment success but not immediate stone clearance or lengthy tries of cannulation. Moreover, wearing uncomfortable personal protective equipment could play a minor, but considerable role when performing time-demanding procedures. Inline, the decrease of successful evacuation of stones taken together with a significant increase of successful implementation of biliary stents could be a result of more conservative treatment of hard-to-evacuate stones in COVID-19 pandemic period.

\section{Summary}

We are aware of several limitations of this study coming from its retrospective character as well as a relatively short period of observation. Nevertheless, here we show a real-life scenario impacting the function of 2 endoscopy units in large-capacity tertiary centers during the early phase of the COVID-19 pandemic. Although notably influenced by the pandemic, tertiary reference centers maintained their function in general, complying with the safety rules, which resulted in the absence of endoscopyrelated infections of staff. A general decrease in performed endoscopies and a devastating influence on endoscopy training needs urgent and adaptive solutions. Multicenter, prospective studies concerning local healthcare policies are urgently needed to establish suitable pro- tocols and precise guidelines for safe and efficient practicing of endoscopy in the COVID-19 pandemic and postpandemic period.

\section{Acknowledgement}

The authors would like to thank Prof. Jörg Schirra for his kind help in data acquisition.

\section{Statement of Ethics}

The study was approved by the Ethics Committee at LMU Munich (Project No. 20-379).

\section{Conflict of Interest Statement}

The authors declare no potential conflicts of interest concerning the research, authorship, and/or publication of this article.

\section{Funding Sources}

M.Z. resident at LMU Munich is supported by the United European Gastroenterology Research Fellowship. C.S. is supported by DZIF grants (DZIF TTU06.714_00, DZIF TTU06.827_00). S.S. was funded by the Association for the promotion of science and research at the medical faculty of the Ludwig Maximillian's University Munich.

\section{Authors Contributions}

J.M., C.S., M.Z., and S.S. designed the study. M.Z., S.S., M.C., P.W., and M.H. acquired data. U.M. analyzed and interpreted data. J.M. and C.S. critically revised the manuscript. All the authors approved the final version of the manuscript.

\section{References}

1 Sultan S, Lim JK, Altayar O, Davitkov P, Feuerstein JD, Siddique SM, et al. AGA institute rapid recommendations for gastrointestinal procedures during the COVID-19 pandemic. Gastroenterology. 2020;159(2):73958.e4.

2 Gralnek IM, Hassan C, Beilenhoff U, Antonelli G, Ebigbo A, Pellisè M, et al. ESGE and ESGENA Position Statement on gastrointestinal endoscopy and the COVID-19 pandemic. Endoscopy. 2020;52(6):483-90.

3 Seda G, Parrish JS. Augmenting citical care capacity in a disaster. Crit Care Clin. 2019; 35(4):563-73.
4 Mahadev S, Aroniadis OS, Barraza L, Agarunov E, Goodman AJ, Benias PC, et al. Impact of the COVID-19 pandemic on endoscopy practice: results of a cross-sectional survey from the New York metropolitan area. Gastrointest Endosc. 2020;92(3):788-9.

5 Schmiderer A, Schwaighofer H, Niederreiter $\mathrm{L}$, et al. Decline in acute upper gastrointestinal bleeding during Covid-19 pandemic after lockdown in Austria. Endoscopy. 2020; 52(11):1036-8.
6 The Johns Hopkins Universiy \& Medicine Coronavirus Resource Centre. Available from: https://coronavirus.jhu.edu/data\#charts.

7 WHO Director-General's opening remarks at the media briefing on COVID-19. Available from: https://www.who.int/dg/speeches/detail/who-directorgeneral-s-opening-remarks-at-the-media-briefing-on-covid-19 Accessed 2020 March 11.

8 Neumann WL, Luján GM, Genta RM. Gastric heterotopia in the proximal oesophagus ("inlet patch"): Association with adenocarcinomas arising in Barrett mucosa. Dig Liver Dis. 2012;44(4):292-6.
Influence of COVID-19 Pandemic on Endoscopic Procedures 
9 Peitz U, Vieth M, Evert M, Arand J, Roessner A, Malfertheiner P. The prevalence of gastric heterotopia of the proximal esophagus is underestimated, but preneoplasia is rare - correlation with Barrett's esophagus. BMC Gastroenterol. 2017;17(1):87.

10 Bajbouj M, Meining A, Schmid RM. Endoscopic diagnosis and treatment of inlet patch: justification, techniques, and results. Tech Gastrointest Endosc. 2014;16(1):4952.

11 Rex DK, Bond JH, Winawer S, Levin TR, Burt RW, Johnson DA, et al. Quality in the technical performance of colonoscopy and the continuous quality improvement process for colonoscopy: recommendations of the U.S. Multi-Society Task Force on Colorectal Cancer. Am J Gastroenterol. 2002;97(6):1296308.

12 Marshall JB, Barthel JS. The frequency of total colonoscopy and terminal ileal intubation in the 1990s. Gastrointest Endosc. 1993;39(4): 518-20.

13 Testoni PA, Mariani A, Aabakken L, Arvanitakis $\mathrm{M}$, Bories $\mathrm{E}$, Costamagna $\mathrm{G}$, et al. Papillary cannulation and sphincterotomy techniques at ERCP: European Society of Gastrointestinal Endoscopy (ESGE) Clinical Guideline. Endoscopy. 2016;48(07): 657-83.
14 Domagk D, Oppong KW, Aabakken L, Czakó L, Gyökeres T, Manes G, et al. Performance measures for ERCP and endoscopic ultrasound: a European Society of Gastrointestinal Endoscopy (ESGE) Quality Improvement Initiative. Endoscopy. 2018;50(11):1116-27.

15 Pawlak KM, Kral J, Khan R, Amin S, Bilal M, Lui RN, et al. Impact of COVID-19 on endoscopy trainees: an international survey. Gastrointest Endosc. 2020;92(20):925-35.

16 Garbe J, Eisenmann S, Walter S, Lammert F, Darwiche K, Rosendahl J. German endoscopy unit preparations for the COVID-19 pandemic: A nationwide survey. Gastroenterology;159(2):778-80. 